\title{
Effect of choice of treatment modality on the incidence of shunt-dependent hydrocephalus after aneurysmal subarachnoid hemorrhage
}

\author{
Masaomi Koyanagi, MD, PhD, ${ }^{1}$ Hitoshi Fukuda, MD, PhD, ${ }^{2}$ Masaaki Saiki, MD, PhD, ${ }^{3}$ \\ Yoshihito Tsuji, MD, PhD, ${ }^{3}$ Benjamin Lo, MD, PhD, ${ }^{4}$ Toshinari Kawasaki, MD, ${ }^{2}$ Yoshihiko loroi, MD, \\ Ryu Fukumitsu, MD, PhD, ${ }^{3}$ Ryota Ishibashi, MD, ${ }^{2}$ Masashi Oda, MD, PhD, ${ }^{1}$ \\ Osamu Narumi, MD, PhD, ${ }^{1}$ Masaki Chin, MD, ${ }^{2}$ Sen Yamagata, MD, PhD, ${ }^{2}$ and \\ Susumu Miyamoto, MD, $\mathrm{PhD}^{5}$
}

1Department of Neurosurgery, National Hospital Organization Himeji Medical Center, Himeji; ${ }^{2}$ Department of Neurosurgery, Kurashiki Central Hospital, Kurashiki; ${ }^{3}$ Department of Neurosurgery, Otsu Red Cross Hospital, Otsu; ${ }^{5}$ Department of Neurosurgery, Kyoto University Graduate School of Medicine, Kyoto, Japan; and 4Department of Neurology and Neurosurgery, Montreal Neurological Institute and Hospital, McGill University Health Centre, Montreal, Quebec, Canada

OBJECTIVE Shunt-dependent hydrocephalus (SDHC) may arise after aneurysmal subarachnoid hemorrhage (aSAH) as CSF resorptive mechanisms are disrupted. Using propensity score analysis, the authors aimed to investigate which treatment modality, surgical clipping or endovascular treatment, is superior in reducing rates of SDHC after aSAH.

METHODS The authors' multicenter SAH database, comprising 3 stroke centers affiliated with Kyoto University, Japan, was used to identify patients treated between January 2009 and July 2016. Univariate and multivariate analyses were performed to characterize risk factors for SDHC after aSAH. A propensity score model was generated for both treatment groups, incorporating relevant patient covariates to detect any superiority for prevention of SDHC after aSAH.

RESULTS A total of 566 patients were enrolled in this study. SDHC developed in 127 patients (22\%). On multivariate analysis, age older than 53 years, the presence of intraventricular hematoma, and surgical clipping as opposed to endovascular coiling were independently associated with SDHC after aSAH. After propensity score matching, 136 patients treated with surgical clipping and 136 with endovascular treatment were matched. Propensity score-matched cohorts exhibited a significantly lower incidence of SDHC after endovascular treatment than after surgical clipping ( $16 \%$ vs $30 \%$, $p=0.009$; OR 2.2, 95\% Cl 1.2-4.2). SDHC was independently associated with poor neurological outcomes (modified Rankin Scale score 3-6) at discharge (OR 4.3, 95\% Cl 2.6-7.3; $p<0.001$ ).

CONCLUSIONS SDHC after aSAH occurred significantly more frequently in patients who underwent surgical clipping. Strategies for treatment of ruptured aneurysms should be used to mitigate SDHC and minimize poor outcomes.

https://thejns.org/doi/abs/10.3171/2017.9.JNS171806

KEY WORDS hydrocephalus; cerebrospinal fluid shunt; subarachnoid hemorrhage; propensity score; vascular disorders

$\mathrm{B}$ LOCKAGE of CSF resorptive mechanisms after aneurysmal subarachnoid hemorrhage (aSAH) may lead to hydrocephalus. Permanent CSF diversion, such as ventriculoperitoneal shunting, is required in $11 \%-31 \%$ of aSAH patients due to hydrocephalus. ${ }^{1,8-10,22,25,28,29}$ However, shunt procedures can be associated with high rates of revision $(13 \%-32 \%)^{12,18,24}$ due to complications such as mechanical obstruction, under- or overdrainage, and infection, with associated extended length of hospital stay and patient morbidity. ${ }^{23}$

To date, it is unclear whether surgical clipping or endovascular coiling is associated with higher incidences

ABBREVIATIONS ACA = anterior cerebral artery; aSAH = aneurysmal subarachnoid hemorrhage; ICA = internal carotid artery; ICH = intracerebral hemorrhage; IQR = interquartile range; IVH = intraventricular hemorrhage; $\mathrm{MCA}=$ middle cerebral artery; SDHC = shunt-dependent hydrocephalus; WFNS = World Federation of Neurosurgical Societies.

SUBMITTED July 24, 2017. ACCEPTED September 25, 2017.

INCLUDE WHEN CITING Published online March 9, 2018; DOI: 10.3171/2017.9.JNS171806. 
of shunt-dependent hydrocephalus (SDHC). The patient cohort of 1448 patients derived from the Japanese Stroke DataBank revealed that endovascular coiling is associated with lower rates of post-aSAH hydrocephalus. ${ }^{28}$ Yet, recent retrospective analysis of the BRAT (Barrow Ruptured Aneurysm Trial) database showed no significant differences in shunt placement rates between the treatment modalities. ${ }^{29}$

To attempt to overcome the study limitation of selection bias in these retrospective studies, we used propensity score analysis to identify aSAH patient cohorts of similar clinical characteristics from a prospectively maintained multicenter aSAH database. We characterized the incidences of post-aSAH hydrocephalus requiring shunt placement between patients who underwent clipping and those who underwent coiling.

\section{Methods}

This study is based on criteria from the STROBE (Strengthening the Reporting of Observational Study in Epidemiology) statement. ${ }^{26}$ The study protocol was approved by the institutional review board of the National Hospital Organization Himeji Medical Center, and a waiver of consent was sought and obtained for this observational study with no unique patient identifiers.

\section{Data Source}

Between January 2009 and July 2016, patients who underwent surgical clipping and endovascular treatment for ruptured cerebral aneurysms within 72 hours after onset were entered into a prospectively maintained multicenter SAH database comprising 3 Japanese stroke centers: Himeji Medical Center, Kurashiki Central Hospital, and Otsu Red Cross Hospital. Variables collected in this database included age (years), sex (male or female), World Federation of Neurosurgical Societies (WFNS) grade on hospital admission, Hijdra scale for evaluation of subarachnoid clot burden (0-13), ${ }^{13}$ presence of intracerebral hemorrhage (ICH), presence of intraventricular hemorrhage (IVH), aneurysm characteristics such as maximum size $(\mathrm{mm})$ and location (anterior cerebral artery [ACA], including the anterior communicating artery, internal carotid artery [ICA]-posterior communicating artery bifurcation and other ICA bifurcation aneurysms, middle cerebral artery $[\mathrm{MCA}]$, or posterior circulation [posterior cerebral artery, basilar tip, basilar artery-superior cerebellar artery bifurcation, and vertebral artery-posterior inferior cerebellar artery bifurcation]), treatment modality for aneurysms (clipping or coiling), presence of acute hydrocephalus (defined as CSF drainage before aneurysm repair), presence of CSF drainage after aneurysm repair, and presence of symptomatic cerebral vasospasm. Demographic information also included date of hospital admission, initial procedure, and date of discharge.

\section{Study Population}

This study included data from consecutive patients with SAH who underwent aneurysm repair by clipping or endovascular treatment for ruptured cerebral aneurysms within 72 hours. Exclusion criteria were 1) dissecting, fu- siform, and mycotic aneurysms because they have different pathological characteristics from saccular aneurysms; and 2) patients who died within 1 month after ictus because of a poor presentation grade.

\section{Management of Subarachnoid Hemorrhage}

Treatment choice, surgical clipping or endovascular coiling, was determined according to multidisciplinary discussions involving both surgical and endovascular teams. Patients presenting with altered mental status and radiological evidence of acute hydrocephalus underwent immediate CSF diversion via external ventriculostomy before aneurysm repair. After securing the aneurysm, in the absence of any contraindications, all patients received prophylactic intravenous infusion of fasudil hydrochloride, were maintained euvolemic to prevent cerebral vasospasm, and had CSF drainage as early as possible to evacuate the subarachnoid blood clot burden. We used intermittent CSF drainage when intracranial pressure reached a predefined threshold. The management protocol was similar among our 3 stroke centers.

\section{Definition of Outcome Variable}

SDHC was defined as hydrocephalus after aSAH requiring shunt diversion surgery. The indication for CSF diversion surgery in SAH patients was based on the occurrence of a clinical constellation of decreased mental status, axial rigidity and/or incontinence beyond the timing of vasospasm, and radiological evidence of enlarged ventricles. Clinical indications for shunting were similar across all 3 stroke centers.

\section{Statistical Analysis}

Commercially available software (version 23, IBM SPSS, IBM Corp.) was used for all statistical analyses. Data are presented as the median and interquartile range (IQR) or the number and percentage of patients. The Mann-Whitney U-test was used for continuous variables, and the Fisher exact test for categorical variables. We analyzed the incidence of SDHC, as well as associated clinical factors. Receiver operating characteristic curve analysis was used to determine the optimal cutoff values of age, Hijdra score, and maximum aneurysm size to predict SDHC. All categorical variables with $\mathrm{p}<0.15$ in univariate analysis were subsequently included in multivariate logistic regression analyses; $\mathrm{p}<0.05$ was considered statistically significant.

\section{Propensity Score Matching Analysis}

Primary outcomes included differences in incidences of SDHC between patients who underwent clipping and those who underwent coil embolization. Since patients in this study were not randomized to clipping or coiling, propensity score matching was performed, with the aneurysm-securing procedure as the dependent variable in order to decrease variability between treatment cohorts. Patients were matched for age, sex, admission WFNS grade, Hijdra score, presence of ICH, IVH, aneurysm, location (ACA, MCA, ICA, or posterior circulation), aneurysm size, and presence of acute hydrocephalus, defined as 
a requirement for CSF drainage prior to aneurysm repair. These variables were selected, as they have previously been associated with SDHC or were deemed a priori to be important demographic characteristics. After propensity score generation, cohort and treatment groups underwent 1:1 nearest-neighbor matching of the propensity score with a caliper width of 0.2 of the standard deviation of the score. ${ }^{4}$ Matching was performed without replacement treatment, and control units not meeting matching criteria were excluded. We used the standardized difference to measure covariate balance. Imbalance of covariate was defined as absolute value greater than $1.96 \times \sqrt{ }(2 / \mathrm{n} 3)$.

\section{Results}

A total of 621 consecutive patients who underwent aneurysm repair via surgical clipping or endovascular treatment within 72 hours after ictus at Himeji Medical Center, Kurashiki Central Hospital, and Otsu Red Cross Hospital were registered into our database (January 2009 to July 2016). Three hundred twenty-six patients were treated at Kurashiki Central Hospital, 178 patients at Otsu Red Cross Hospital, and 117 patients at Himeji Medical Center. Of the 621 patients, 32 patients with dissecting aneurysms and 23 patients who died within 1 month due to poorgrade SAH were excluded. As a result, 566 patients (91\%) were enrolled in this study.

The median patient age was 65 years (IQR 53-75 years), and 422 patients (75\%) were female. Thirty-three percent of patients had an WFNS grade of IV or V on presentation, and 92\% ( $\mathrm{n}=519)$ of the patient cohort had anterior circulation aneurysms. A total of 382 patients (67\%) had their ruptured aneurysms repaired by surgical clipping (Table 1).

SDHC developed in 127 patients (22\%) in our cohort. On univariate analysis, patients with SDHC were older (median age 70 vs 63 years, $\mathrm{p}<0.001$ ), and were more likely to 1) present with poor neurological grade (WFNS grade IV or V: $50 \%$ vs $28 \%$, p < 0.001 ; OR $2.7,95 \%$ CI 1.7-4.1), 2) have increased subarachnoid clot burden with higher Hijdra scores (median 22 vs 17, $\mathrm{p}<0.001), 3$ ) experience acute hydrocephalus ( $37 \%$ vs $25 \% \mathrm{p}=0.013$; OR $1.7,95 \%$ CI 1.1-2.7), 4) have an IVH (62\% vs $34 \%$, p < 0.001; OR 3.2, 95\% 2.1-4.9), and 5) have an associated ICH (31\% vs $18 \%, \mathrm{p}=0.002$; OR $2.1,95 \%$ CI $1.2-3.4)$. In addition, SDHC occurred significantly more often in patients who underwent clipping than in those who underwent coiling ( $78 \%$ vs $64 \%, \mathrm{p}=0.005$; OR $1.9,95 \%$ CI 1.2-3.2). The incidence of SDHC was also increased in patients who required postoperative CSF drainage, compared with those who did not require such drainage $(93 \%$ vs $83 \%, \mathrm{p}=0.004$; OR 2.7, 95\% CI 1.3-6.3; Table 1).

Thereafter, we determined the optimal cutoff values for age and Hijdra score using receiver operating characteristic curve analysis. For age, the optimal cutoff value to detect the development of SDHC was 53 years, with sensitivity and specificity of 0.93 and 0.29 , respectively. The optimal cutoff value for Hijdra score was 19, with sensitivity and specificity of 0.67 and 0.55 , respectively. On multivariate logistic regression analysis, age older than 53 years, presence of IVH, and surgical clipping, as opposed
TABLE 1. Baseline, clinical, and procedure-related characteristics of patients and univariate analysis for predictors of SDHC

\begin{tabular}{|c|c|c|c|c|}
\hline Variable & Total & SDHC & $\begin{array}{l}\text { No } \\
\text { SDHC }\end{array}$ & $\begin{array}{c}p \\
\text { Value }\end{array}$ \\
\hline No. of patients & 566 & 127 & 439 & \\
\hline $\begin{array}{l}\text { Median age in yrs } \\
(\mathrm{IQR})\end{array}$ & $65(53-75)$ & $70(61-77)$ & $63(51-74)$ & $<0.001$ \\
\hline Female sex & $422(75)$ & $95(75)$ & $327(74)$ & 1 \\
\hline WFNS grade IV or V & $185(33)$ & $64(50)$ & $121(28)$ & $<0.001$ \\
\hline $\begin{array}{l}\text { Median aneurysm size } \\
\text { in } \mathrm{mm}(\mathrm{IQR})\end{array}$ & $5(4-7)$ & $5(4-7)$ & $5(4-7)$ & 0.96 \\
\hline \multicolumn{5}{|l|}{ Aneurysm location } \\
\hline ACA & $196(35)$ & $53(42)$ & $143(33)$ & 0.058 \\
\hline ICA & $162(29)$ & $33(26)$ & $129(29)$ & 0.50 \\
\hline MCA & $161(28)$ & $34(27)$ & $127(29)$ & 0.66 \\
\hline Posterior circulation & $47(8)$ & $7(6)$ & $40(9)$ & 0.27 \\
\hline $\begin{array}{l}\text { Median Hijdra score } \\
\qquad(\mathrm{IQR})\end{array}$ & $18(10-24)$ & $22(14-27)$ & $17(9-23)$ & $<0.001$ \\
\hline IVH & $229(40)$ & $79(62)$ & $150(34)$ & $<0.001$ \\
\hline $\mathrm{ICH}$ & $120(21)$ & $40(31)$ & $80(18)$ & 0.002 \\
\hline Acute hydrocephalus & $158(28)$ & $47(37)$ & $111(25)$ & 0.013 \\
\hline $\begin{array}{l}\text { Symptomatic vaso- } \\
\text { spasm }\end{array}$ & $88(16)$ & $30(24)$ & $58(13)$ & 0.008 \\
\hline Surgical clipping & 382 (67) & $99(78)$ & $283(64)$ & 0.005 \\
\hline Postop CSF drainage & $482(85)$ & $118(93)$ & $364(83)$ & 0.004 \\
\hline
\end{tabular}

Unless stated otherwise, values are expressed as number of patients (\%).

to endovascular coiling, were independently associated with SDHC (Table 2).

To account for patient nonrandomization in their respective aneurysm treatment arms, we performed propensity score matching with the aim of comparing similar patient subgroups during analysis and to control for covariate differences. Before propensity score matching, analysis of patient baseline characteristics revealed that those who underwent surgical clipping were 1) younger (median age 63 vs 69 years, $\mathrm{p}<0.001$ ), 2) less likely to be female ( $71 \%$ vs $82 \%, p=0.005), 3$ ) more likely to have smaller aneurysms (median size 5.0 vs $5.8 \mathrm{~mm}, \mathrm{p}=0.04$ ),

TABLE 2. Multivariate analyses for predictors of SDHC

\begin{tabular}{lccc}
\hline \multicolumn{1}{c}{ Variable } & OR & $95 \% \mathrm{Cl}$ & $\mathrm{p}$ Value \\
\hline Age $>53$ yrs & 4.3 & $2.1-8.9$ & $<0.001$ \\
\hline WFNS grade IV or V & 1.5 & $0.9-2.5$ & 0.11 \\
\hline ACA aneurysms & 1.4 & $0.9-2.3$ & 0.11 \\
\hline Hijdra score $>19$ & 1.4 & $0.8-2.2$ & 0.21 \\
\hline IVH & 2.2 & $1.4-3.5$ & $<0.001$ \\
\hline ICH & 1.4 & $0.8-2.4$ & 0.25 \\
\hline Acute hydrocephalus & 1.2 & $0.7-1.9$ & 0.48 \\
\hline Symptomatic vasospasm & 1.5 & $0.9-2.6$ & 0.14 \\
\hline Surgical clipping & 1.9 & $1.2-3.2$ & 0.011 \\
\hline Postop CSF drainage & 2.0 & $0.9-2.5$ & 0.082 \\
\hline
\end{tabular}


TABLE 3. Patient demographics and clinical and radiological characteristics of 566 unmatched patients

\begin{tabular}{lccc}
\hline \multicolumn{1}{c}{ Variable } & $\begin{array}{c}\text { Surgical } \\
\text { Clipping }\end{array}$ & $\begin{array}{c}\text { Endovascular } \\
\text { Treatment }\end{array}$ & $\begin{array}{c}\mathrm{p} \\
\text { Value }\end{array}$ \\
\hline No. of patients & 382 & 184 & \\
\hline Median age in yrs (IQR) & $63(52-73)$ & $69(57-80)$ & $<0.001$ \\
\hline Female sex & $271(71)$ & $151(82)$ & 0.005 \\
\hline WFNS grade IV or V & $123(32)$ & $62(34)$ & 0.77 \\
\hline $\begin{array}{l}\text { Median aneurysm size in } \\
\text { mm (IQR) }\end{array}$ & $5.0(4.0-7.0)$ & $5.8(4.1-7.0)$ & 0.04 \\
\hline Aneurysm location & & & \\
\hline ACA & $136(36)$ & $60(33)$ & 0.51 \\
\hline ICA & $103(27)$ & $59(32)$ & 0.23 \\
\hline MCA & $140(37)$ & $21(11)$ & $<0.001$ \\
\hline Posterior circulation & $3(0.8)$ & $44(24)$ & $<0.001$ \\
\hline Subarachnoid clot burden & & & \\
\hline Median Hijdra score (IQR) & $19(10-25)$ & $17(8-24)$ & 0.20 \\
\hline ICH & $93(24)$ & $27(15)$ & 0.009 \\
\hline IVH & $154(40)$ & $75(41)$ & 0.93 \\
\hline Acute hydrocephalus & $128(34)$ & $30(16)$ & $<0.001$ \\
\hline
\end{tabular}

Unless otherwise stated, values are expressed as number of patients (\%).

4) more likely to have aneurysms located in the middle cerebral artery (MCA) territory ( $37 \%$ vs $11 \%$, p < 0.001 ), 5) less likely to have aneurysms in the posterior circulatory territory $(0.8 \%$ vs $24 \%, \mathrm{p}<0.001), 6)$ more likely to have an ICH ( $24 \%$ vs $15 \%, \mathrm{p}=0.009)$, and 7 ) more likely to have presented with acute hydrocephalus (34\% vs $16 \%$, $\mathrm{p}<0.001$; Table 3).

The standard deviation of the propensity score was 0.23 . Thus, a caliper width was set at 0.05. After 1:1 matching with a caliper width of 0.05 , equal numbers of clipping and coiling patients ( $n=136$ per group) were matched. Because there were 136 patients in each group, the cutoff value of absolute standardized difference for imbalance was 0.24 . Across the baseline covariates, the absolute standardized differences ranged from a low of 0 to a high of 0.19 , indicating that the means and prevalence of continuous and dichotomous variables were similar between treatment groups in the matched sample (Table 4).

Propensity score-matched cohorts exhibited significantly fewer incidences of SDHC when patients were treated by endovascular procedures than when they were treated by surgical clipping (16\% vs 30\%, p $=0.009$; OR 2.2, 95\% CI 1.2-4.2) (Table 5). Seven patient subgroups exhibited baseline characteristic differences prior to propensity matching. Older age, female sex, aneurysms with larger diameter, and posterior circulation aneurysms were more likely to be treated endovascularly, whereas those with aneurysms located in the MCA territory, $\mathrm{ICH}$, and acute hydrocephalus were more likely to be treated with clipping (Table 3). Analyses of these subgroups revealed that, when treated endovascularly rather than surgically, patients experienced fewer SDHC events if they were older than 53 years (OR 0.48, 95\% CI 0.28-0.79; $\mathrm{p}=0.003$ ), were female (OR 0.57, 95\% CI 0.33-0.97; $\mathrm{p}=0.029)$, harbored aneurysms larger than $3.2 \mathrm{~mm}$ in maximum diameter (OR $0.51,95 \%$ CI $0.30-0.84 ; \mathrm{p}=0.006)$, and had an ICH (OR $0.28,95 \%$ CI $0.065-0.91 ; p=0.022)$. In addition, no significant differences in SDHC events were noted in patients with posterior circulation aneurysms, aneurysms located in the MCA territory, and acute hydrocephalus at presentation.

Compared with matched patients, no match was found for the 294 patients who underwent aneurysm repair within 72 hours and were younger ( 73 vs 76 years, $p=0.004$ ), less likely to be female ( $69 \%$ vs $82 \%, \mathrm{p}=0.001)$, more likely to have smaller aneurysms $(5 \mathrm{~mm}$ vs $6 \mathrm{~mm}, \mathrm{p}=$ 0.001 ), less likely to have aneurysms located in the ICA

TABLE 4. Patient demographics and clinical and radiological characteristics of 272 propensity score-matched patients

\begin{tabular}{|c|c|c|c|c|}
\hline Variable & Surgical Clipping & Endovascular Treatment & $p$ Value & Standardized Difference \\
\hline No. of patients & 136 & 136 & & \\
\hline Median age in yrs (IQR) & $67(55-76)$ & $67(56-78)$ & 0.90 & 0.010 \\
\hline Female sex & $113(83)$ & $107(79)$ & 0.44 & 0.10 \\
\hline WFNS grade IV or V & $38(28)$ & $48(35)$ & 0.24 & 0.15 \\
\hline Median aneurysm size in mm (IQR) & $6.0(4.0-8.0)$ & $5.8(4.8-7.0)$ & 0.94 & 0.011 \\
\hline \multicolumn{5}{|l|}{ Aneurysm location } \\
\hline ACA & $46(34)$ & $58(43)$ & 0.17 & 0.19 \\
\hline ICA & $61(45)$ & $54(40)$ & 0.46 & 0.10 \\
\hline MCA & $26(19)$ & $21(15)$ & 0.52 & 0.11 \\
\hline Posterior circulation & $3(0.2)$ & $3(0.2)$ & $>0.99$ & 0 \\
\hline \multicolumn{5}{|l|}{ Subarachnoid clot burden } \\
\hline Median Hijdra score (IQR) & $19(9-24)$ & $18(9-25)$ & 0.85 & 0.002 \\
\hline $\mathrm{ICH}$ & $24(18)$ & $26(19)$ & 0.88 & 0.026 \\
\hline IVH & $54(40)$ & $51(38)$ & 0.80 & 0.041 \\
\hline Acute hydrocephalus & $24(18)$ & $19(14)$ & 0.51 & 0.11 \\
\hline
\end{tabular}

Unless otherwise stated, values are expressed as number of patients (\%). 
TABLE 5. Outcomes after 1:1 matching by propensity score

\begin{tabular}{ccccc}
\hline Variable & $\begin{array}{c}\text { Surgical Clipping } \\
(\mathrm{n}=136)\end{array}$ & $\begin{array}{c}\text { Endovascular } \\
\text { Treatment }(\mathrm{n}=136)\end{array}$ & $\begin{array}{c}\mathrm{OR} \\
(95 \% \mathrm{Cl})\end{array}$ & $\begin{array}{c}\mathrm{p} \\
\text { Value }\end{array}$ \\
\hline SDHC & $41(30)$ & $22(16)$ & $2.2(1.2-4.2)$ & 0.009 \\
\hline
\end{tabular}

Unless otherwise stated, values are expressed as number of patients (\%).

territory $(0.3 \%$ vs $42 \%, \mathrm{p}<0.001)$, more likely to have aneurysms located in the MCA territory $(39 \%$ vs $17 \%$, p < $0.001)$, more likely to have aneurysms located in the posterior circulation territory ( $14 \%$ vs $2.2 \%, \mathrm{p}<0.001)$, more likely to have presented with acute hydrocephalus $(39 \%$ vs $16 \%, \mathrm{p}<0.001$ ), and more likely to have undergone surgical clipping ( $84 \%$ vs $50 \%, \mathrm{p}<0.001$ ). In addition, on multivariate analysis of unmatched patients, age older than 53 years (OR 5.0,95\% CI 1.9-13; $\mathrm{p}=0.001$ ), presence of IVH (OR 3.0, 95\% CI 1.6-5.4; p < 0.001), and surgical clipping, as opposed to endovascular coiling (OR 2.9, 95\% CI 1.1-7.4; $\mathrm{p}=0.025)$, were independently associated with SDHC after SAH.

We subsequently examined whether SDHCs were independently associated with neurological outcomes after SAH at discharge. The median length of hospital stay in this cohort was 40 days (IQR 27-56 days). On multivariate logistic regression analyses, SDHC was independently associated with poor neurological outcomes (modified Rankin Scale scores 3-6) at discharge (OR 4.3, 95\% CI $2.6-7.3 ; \mathrm{p}<0.001)$. Other clinical covariates associated with poor neurological outcomes included older age (OR $1.1,95 \%$ CI $1.1-1.1 ; \mathrm{p}<0.001$ ), WFNS grade IV or V (OR $4.1,95 \%$ CI 2.4-6.8; $\mathrm{p}<0.001$ ), larger aneurysm size (OR $1.1,95 \%$ CI 1.0-1.2; $\mathrm{p}=0.014)$, and presence of ICH (OR $2.3,95 \%$ CI $1.3-4.1 ; p=0.003)$. Furthermore, patients with SDHC had a significantly longer hospital stay than those without SDHC (median length of hospital stay $73 \mathrm{vs}$ 33 days, $\mathrm{p}<0.001$ ). The frequency of shunt revision was $11 \%$ (14 of 127). Of the 14 patients who underwent shunt revisions, 4 required hospital readmission.

\section{Discussion}

Our study found that SDHC after aSAH occurred significantly more frequently in patients who underwent surgical clipping than in those who underwent endovascular treatment. We performed propensity score adjustment by using clinical covariates to minimize the impact of selection bias on observed treatment outcomes in our analyses. Our results suggest that surgical clipping is associated with a higher incidence of SDHC development after aSAH compared with endovascular treatment. SDHC was also associated with poor neurological outcomes at hospital discharge.

To date, it is unclear which aneurysm-securing modality (clipping or endovascular treatment) can reduce the incidence of SDHC after aSAH. Our multivariate analyses and propensity score matching demonstrated that surgical clipping was associated with a higher incidence of SDHC after SAH compared with endovascular treatment. Our findings are in agreement with those from a recent large registration study involving 1448 patients, which showed that coil embolization, when compared with clipping, is associated with significantly lower risks for development of hydrocephalus after aSAH. ${ }^{28}$ Surgical clipping via a transsylvian approach would entail unilateral arachnoid membrane dissection that may alter CSF flow dynamics. As opposed to open clipping, endovascular treatment may not directly disturb CSF flow dynamics and resorptive mechanisms.

Yet, other reports have made the observation that SDHC rates are not increased after clipping in patient subgroups in which fenestration of the lamina terminalis was also performed. ${ }^{3,16}$ It was once considered that anterior ventriculostomy created by fenestration of lamina terminalis could assist in CSF flow dynamics to decrease the rates of shunt dependence after aSAH. However, the functional patency of such fenestrated lamina terminalis when performed as part of surgical clipping remains uncertain. ${ }^{5}$ Moreover, a systematic review of 11 nonrandomized studies pooling 1973 patients found no significant differences in the incidences of long-term shunt-dependent hydrocephalus in patients who underwent lamina terminalis fenestration compared with those without such fenestration during surgical clipping..$^{17}$ On the basis of this evidence, guidelines for the management of chronic hydrocephalus after aSAH from the American Heart Association recommend that fenestration of the lamina terminalis should not be routinely performed. ${ }^{6}$

Furthermore, our study findings can be contrasted with those from previous studies that have shown no significant differences in the incidences of SDHC between surgical clipping and endovascular treatment. ${ }^{1,8,14,26,29}$ A possible hypothesis to account for this discrepancy is the age differences noted in patient cohorts, with the median age of our cohort being 65 years compared with the median age of 53-55 years in other patient populations that did not exhibit significant differences in secondary hydrocephalus regardless of treatment modalities.1,14,26,29 Prior studies have shown that advanced age is an independent risk factor for SDHC after aSAH. 1,10,14,22,27,28 Older patients are at risk for SDHC secondary to decreased microcirculation across ventricular walls and ventricular enlargement secondary to age-related brain atrophy. In addition, CSF secretion and turnover decrease with age. ${ }^{20}$ Together, larger subarachnoid spaces in older aSAH patients can harbor more subarachnoid clot burden, which may facilitate inflammation and fibrosis, ultimately leading to disruption of CSF flow dynamics. This is supported by our study observation that fewer SDHC events were noted in the subgroup of patients older than 53 years who underwent endovascular treatment.

In the current observational study, we attempted to mitigate the effects of selection bias by using propensity score matching to reduce the effects of potential confounders. On one hand, the process of blinding and randomization in a prospective randomized controlled trial may ensure that enrolled patients in one treatment arm will not systemically differ from those in the other treatment arm in both measured and unmeasured baseline characteristics. On the other hand, the process of blinding and randomization for aneurysm-securing techniques may not be very feasible due to both anatomical and technical considerations, as well as clinical manifestations and evolution of patients' brain-body interactions. ${ }^{19}$ 
This present study also showed that SDHC was an independent risk factor for poor neurological outcome; other risk factors included older age, poor admission neurological grade, large aneurysm size, and presence of ICH. These findings are consistent with those from previous studies showing a relationship between hydrocephalus and poor neurological outcomes.,22 SDHC was also reported to associated with pronounced cognitive deficit and impaired quality of life. ${ }^{15}$ Although clinical improvement after shunt placement was reported in $74.4 \%$ of patients with secondary hydrocephalus, ${ }^{7}$ permanent CSF diversion is known to be associated with complications, including ICH following insertion of a ventricular catheter, infection, malfunction, and obstruction, ${ }^{21}$ leading to frequent shunt revision-associated comorbid states. In the present study, $11 \%$ of shunt-treated patients underwent revision. This rate is comparable with rates reported in the literature, ${ }^{12,18}$ with both medical and economic implications of shunt-related complications. In our study, shunt-treated patients also had significantly longer hospital stays. Although the reasons for such increased duration of hospital stays are multifactorial, extended stay can result in additional health care and health-related quality-of-life costs. We aim to further study risk factors for development of SDHC to avoid them.

Our study has several limitations. First, selection bias exists, as patients were not randomized to clipping or coiling in our retrospective cohort study. Although propensity score adjustments of patient age, sex, and admission status minimized differences between clipping and coiling cohorts, other unmeasured, latent variables and their interactions may have contributed to selection bias. Second, surgical clipping was the preferred modality of treatment in our study. Two-thirds of aSAH patients were treated using surgical clipping, as they were offered such modality of treatment by the treating surgeon according to multidisciplinary discussions involving both surgical and endovascular teams. As a result, after propensity score matching, some clip-treated patients were excluded for further analysis due to dissimilar covariate characteristics. Third, we excluded dissecting aneurysms because they are considered to represent a distinct clinical pathological entity. Finally, our patient cohort's length of hospital stay was longer than that reported in other studies, ${ }^{11}$ with a median length of 40 days. In Japan, our hospitals often provide acute-stage postoperative critical care, subacute stroke care, and early rehabilitation in a single hospitalization. As such, even though longer hospitalizations in our cohort might reflect in-hospital comorbidities, we believe that our continuum of aSAH care can maximize patient functional outcomes and prevent unexpected hospital readmissions. We aim to avoid secondary hydrocephalus as a common cause of hospital readmissions, which may be experienced in as high as $11.4 \%$ of patients who received surgical clipping or endovascular treatment after aSAH. ${ }^{11}$

\section{Conclusions}

SDHC after aSAH was significantly more frequent in patients who underwent surgical clipping and was associated with poor neurological outcomes. Strategies for treatment of ruptured aneurysms should be used to mitigate secondary normal pressure hydrocephalus and maximize long-term neurological and functional outcomes.

\section{Acknowledgments}

We would like to acknowledge and thank Kiyomi Hiraoka for her statistical expertise and assistance in statistical analysis.

\section{References}

1. Adams H, Ban VS, Leinonen V, Aoun SG, Huttunen J, Saavalainen T, et al: Risk of shunting after aneurysmal subarachnoid hemorrhage: a collaborative study and initiation of a consortium. Stroke 47:2488-2496, 2016

2. Akyuz M, Tuncer R: The effects of fenestration of the interpeduncular cistern membrane arousted to the opening of lamina terminalis in patients with ruptured ACoA aneurysms: a prospective, comparative study. Acta Neurochir (Wien) 148:725-732, 2006

3. Andaluz N, Zuccarello M: Fenestration of the lamina terminalis as a valuable adjunct in aneurysm surgery. Neurosurgery 55:1050-1059, 2004

4. Austin PC: Optimal caliper widths for propensity-score matching when estimating differences in means and differences in proportions in observational studies. Pharm Stat 10:150-161, 2011

5. Chohan MO, Carlson AP, Hart BL, Yonas H: Lack of functional patency of the lamina terminalis after fenestration following clipping of anterior circulation aneurysms. J Neurosurg 119:629-633, 2013

6. Connolly ES Jr, Rabinstein AA, Carhuapoma JR, Derdeyn CP, Dion J, Higashida RT, et al: Guidelines for the management of aneurysmal subarachnoid hemorrhage: a guideline for healthcare professionals from the American Heart Association/American Stroke Association. Stroke 43:1711-1737, 2012

7. Daou B, Klinge P, Tjoumakaris S, Rosenwasser RH, Jabbour P: Revisiting secondary normal pressure hydrocephalus: does it exist? A review. Neurosurg Focus 41(3):E6, 2016

8. de Oliveira JG, Beck J, Setzer M, Gerlach R, Vatter H, Seifert $\mathrm{V}$, et al: Risk of shunt-dependent hydrocephalus after occlusion of ruptured intracranial aneurysms by surgical clipping or endovascular coiling: a single-institution series and metaanalysis. Neurosurgery 61:924-934, 2007

9. Dehdashti AR, Rilliet B, Rüfenacht DA, de Tribolet N: Shunt-dependent hydrocephalus after rupture of intracranial aneurysms: a prospective study of the influence of treatment modality. J Neurosurg 101:402-407, 2004

10. Dorai Z, Hynan LS, Kopitnik TA, Samson D: Factors related to hydrocephalus after aneurysmal subarachnoid hemorrhage. Neurosurgery 52:763-771, 2003

11. Greenberg JK, Washington CW, Guniganti R, Dacey RG Jr, Derdeyn CP, Zipfel GJ: Causes of 30-day readmission after aneurysmal subarachnoid hemorrhage. J Neurosurg 124:743-749, 2016

12. Hayhurst C, Beems T, Jenkinson MD, Byrne P, Clark S, Kandasamy J, et al: Effect of electromagnetic-navigated shunt placement on failure rates: a prospective multicenter study. $\mathbf{J}$ Neurosurg 113:1273-1278, 2010

13. Hijdra A, Brouwers PJ, Vermeulen M, van Gijn J: Grading the amount of blood on computed tomograms after subarachnoid hemorrhage. Stroke 21:1156-1161, 1990

14. Hoh BL, Kleinhenz DT, Chi YY, Mocco J, Barker FG II: Incidence of ventricular shunt placement for hydrocephalus with clipping versus coiling for ruptured and unruptured cerebral aneurysms in the Nationwide Inpatient Sample database: 2002 to 2007. World Neurosurg 76:548-554, 2011

15. Hütter BO, Gilsbach JM: Short- and long-term neurobehavioral effects of lumbar puncture and shunting in patients with 
malabsorptive hydrocephalus after subarachnoid haemorrhage: an explorative case study. J Clin Neurosci 36:88-93, 2017

16. Komotar RJ, Hahn DK, Kim GH, Khandji J, Mocco J, Mayer SA, et al: The impact of microsurgical fenestration of the lamina terminalis on shunt-dependent hydrocephalus and vasospasm after aneurysmal subarachnoid hemorrhage. Neurosurgery 62:123-134, 2008

17. Komotar RJ, Hahn DK, Kim GH, Starke RM, Garrett MC, Merkow MB, et al: Efficacy of lamina terminalis fenestration in reducing shunt-dependent hydrocephalus following aneurysmal subarachnoid hemorrhage: a systematic review. Clinical article. J Neurosurg 111:147-154, 2009

18. Lee L, King NK, Kumar D, Ng YP, Rao J, Ng H, et al: Use of programmable versus nonprogrammable shunts in the management of hydrocephalus secondary to aneurysmal subarachnoid hemorrhage: a retrospective study with cost-benefit analysis. J Neurosurg 121:899-903, 2014

19. Lo BW, Fukuda H, Nishimura Y, Macdonald RL, Farrokhyar F, Thabane L, et al: Pathophysiologic mechanisms of brainbody associations in ruptured brain aneurysms: a systematic review. Surg Neurol Int 6:136, 2015

20. May C, Kaye JA, Atack JR, Schapiro MB, Friedland RP, Rapoport SI: Cerebrospinal fluid production is reduced in healthy aging. Neurology 40:500-503, 1990

21. McGovern RA, Kelly KM, Chan AK, Morrissey NJ, McKhann GM II: Should ventriculoatrial shunting be the procedure of choice for normal-pressure hydrocephalus? J Neurosurg 120:1458-1464, 2014

22. O'Kelly CJ, Kulkarni AV, Austin PC, Urbach D, Wallace MC: Shunt-dependent hydrocephalus after aneurysmal subarachnoid hemorrhage: incidence, predictors, and revision rates. Clinical article. J Neurosurg 111:1029-1035, 2009

23. Patwardhan RV, Nanda A: Implanted ventricular shunts in the United States: the billion-dollar-a-year cost of hydrocephalus treatment. Neurosurgery 56:139-145, 2005

24. Reddy GK, Bollam P, Shi R, Guthikonda B, Nanda A: Management of adult hydrocephalus with ventriculoperitoneal shunts: long-term single-institution experience. Neurosurgery 69:774-781, 2011

25. Rincon F, Gordon E, Starke RM, Buitrago MM, Fernandez A, Schmidt JM, et al: Predictors of long-term shunt-dependent hydrocephalus after aneurysmal subarachnoid hemorrhage. Clinical article. J Neurosurg 113:774-780, 2010
26. von Elm E, Altman DG, Egger M, Pocock SJ, Gøtzsche PC, Vandenbroucke JP: The Strengthening the Reporting of Observational Studies in Epidemiology (STROBE) statement: guidelines for reporting observational studies. Lancet 370:1453-1457, 2007

27. Wilson CD, Safavi-Abbasi S, Sun H, Kalani MY, Zhao YD, Levitt MR, et al: Meta-analysis and systematic review of risk factors for shunt dependency after aneurysmal subarachnoid hemorrhage. J Neurosurg 126:586-595, 2017

28. Yamada S, Ishikawa M, Yamamoto K, Ino T, Kimura T, Kobayashi S: Aneurysm location and clipping versus coiling for development of secondary normal-pressure hydrocephalus after aneurysmal subarachnoid hemorrhage: Japanese Stroke DataBank. J Neurosurg 123:1555-1561, 2015

29. Zaidi HA, Montoure A, Elhadi A, Nakaji P, McDougall CG, Albuquerque FC, et al: Long-term functional outcomes and predictors of shunt-dependent hydrocephalus after treatment of ruptured intracranial aneurysms in the BRAT trial: revisiting the clip vs coil debate. Neurosurgery 76:608-614, 2015

\section{Disclosures}

The authors report no conflict of interest concerning the materials or methods used in this study or the findings specified in this paper.

\section{Author Contributions}

Conception and design: Koyanagi. Acquisition of data: Koyanagi, Fukuda, Saiki, Tsuji, Kawasaki, Fukumitsu. Analysis and interpretation of data: Koyanagi. Drafting the article: Koyanagi, Lo. Critically revising the article: Fukuda, Saiki, Tsuji, Lo, Miyamoto. Reviewed submitted version of manuscript: Fukuda, Saiki, Tsuji, Lo, Kawasaki, Ioroi, Fukumitsu, Ishibashi, Oda, Narumi, Chin, Yamagata, Miyamoto. Statistical analysis: Koyanagi, Lo. Study supervision: Narumi, Chin, Yamagata, Miyamoto.

\section{Correspondence}

Masaomi Koyanagi: National Hospital Organization Himeji Medical Center, Himeji, Japan. koyanagm@gmail.com. 